\title{
Radioprotective Effect of Epigallocatechin-3-Gallate on Salivary Gland Dysfunction After Radioiodine Ablation in a Murine Model
}

\author{
Jeong-Seok Choi ${ }^{1} \cdot$ Hye-Young An ${ }^{1} \cdot$ In Suh Park² $\cdot$ Seok-Ki Kim³ $\cdot$ Young-Mo Kim¹ $\cdot$ Jae-Yol Lim \\ Departments of ${ }^{1}$ Otorhinolaryngology and ${ }^{2}$ Pathology, Inha University School of Medicine, Incheon; \\ ${ }^{3}$ Department of Nuclear Medicine, National Cancer Center, Goyang, Korea
}

Objectives. Radioiodine (RI) therapy is known to subject cellular components of salivary glands (SG) to oxidative stress leading to SG dysfunction. However, the protective effects of antioxidants on RI-induced SG damage have not been well investigated. The authors investigated the morphometric and functional effects of epigallocatechin-3-gallate (EGCG) administered prior to RI therapy and compared this with the effects of amifostine (a well-known antioxidant) in a murine model of RI sialadenitis.

Methods. Four-week-old female C57BL/6 mice $(\mathrm{n}=48)$ were divided into four groups; a normal control group, a RI-treated group $(0.01 \mathrm{mCi} / \mathrm{g}$ mouse, orally), an EGCG and RI-treated group, and an amifostine and RI-treated group. Animals in these groups were divided into 3 subgroups and euthanized at 15, 30, and 90 days post-RI treatment. Salivary flow rates and lag times were measured, and morphologic and histologic examinations and TUNEL (terminal deoxynucleotidyl transferase biotin-dUDP nick end labeling) assays were performed. Changes in salivary ${ }^{99 \mathrm{~m}}$ Tc pertechnetate uptake and excretion were followed by single-photon emission computed tomography.

Results. Salivary flow rates and lag times to salivation in the EGCG or amifostine groups were better than in the RI-treated group. Histologic examinations of SGs in the EGCG or amifostine group showed more mucin-rich parenchyma and less periductal fibrosis than in the RI-treated group. Fewer apoptotic cells were observed in acini, ducts, and among endothelial cells in the EGCG or amifostine group than in the RI group. In addition, patterns of ${ }^{99 \mathrm{~m}} \mathrm{Tc}$ pertechnetate excretion were quite different in the EGCG or amifostine group than in the RI group.

Conclusion. EGCG supplementation before RI therapy could protect from RI-induced SG damage in a manner comparable to amifostine, and thus, offers a possible means of preventing SG damage by RI.

Keywords. Radiation; Salivary Glands; Thyroid Neoplasms; Tomography, Emission-Computed, Single-Photon; Models, Animal

\section{INTRODUCTION}

Radioiodine (RI) ablation is commonly performed to remove remaining thyroid tissue in patients that have undergone total thy-

\footnotetext{
- Received June 22, 2015

Revised July 27, 2015

Accepted August 17, 2015

- Corresponding author: Jae-Yol Lim

Department of Otorhinolaryngology-Head and Neck Surgery, Inha

University Hospital, Inha University School of Medicine, 27 Inhang-ro, Jung-gu, Incheon 22332, Korea

Tel: +82-32-890-3570, Fax: +82-32-890-3580

E-mail: jylim@inha.ac.kr
}

roidectomy for differentiated thyroid cancer. However, after RI therapy, patients often complain of painful salivary gland (SG) swelling, xerostomia, taste alterations, and oral infections. RI-induced sialadenitis has been reported to occur in $2 \%$ to $67 \%$ of patients that undergo RI therapy, and thus, this condition subsequently diminishes quality of life for many thyroid cancer patients [1].

It is known that radiation damages DNA by producing free radicals, and thus, free radical scavengers can be used to protect tissues [2]. Amifostine is the only radioprotective drug that acts by scavenging free radicals and accumulates in SGs, but it does not protect tumor cells [3,4]. Amifostine can be used in patients

Copyright @ 2016 by Korean Society of Otorhinolaryngology-Head and Neck Surgery.

This is an open-access article distributed under the terms of the Creative Commons Attribution Non-Commercial License (http://creativecommons.org/licenses/by-nc/4.0)

which permits unrestricted non-commercial use, distribution, and reproduction in any medium, provided the original work is properly cited. 
with head and neck cancer undergoing external radiation therapy, but has severe adverse effects that sometimes result in discontinuation. Limited studies have reported on the use of amifostine to protect against internal RI exposure in differentiated thyroid carcinoma, but results vary $[5,6]$.

The efficacies of many natural antioxidants are being investigated in terms of protection against radiation-induced tissue damage, and one of these, epigallocatechin-3-gallate (EGCG) is known to have various beneficial effects, such as, radioprotective [7-9], chemo preventive, antiapoptotic, and anti-inflammatory effects [10-12]. Although RI therapy is known to induce oxidative stress affecting the cellular components of SGs, its protective effects on RI-induced SG damage have not been well investigated. In this study, we investigated the morphometric and functional effects of EGCG administered prior to RI therapy and compared this with the effects of amifostine (a well-known antioxidant) in a murine model of RI sialadenitis.

\section{MATERIALS AND METHODS}

\section{Animal studies}

Sixty-four female, 4-week-old, C57BL/6 mice weighing 18-22 g obtained from the Animal House Facility, International Cancer Research Centre (Korea), were maintained under controlled temperature/light conditions in an animal house with free access to water and a standard mouse diet. Animal studies were performed in compliance with guidelines issued by the International Cancer Research Centre Institutional Animal Ethics Committee. Animals were divided into the following 4 groups (12 animals per group): group I, the normal control; group II, RI exposed group $\left(0.01 \mathrm{mCi} / \mathrm{g}\right.$ body weight, ${ }^{131} \mathrm{I}$; New Korea Industrial, Seoul, Korea, orally); group III, EGCG (40 mg/kg; Santa Cruz, CA, USA, intraperitoneally [i.p.]) 6 hours and 30 minutes before RI exposure; and group IV, administration of amifostine (200 mg/kg; TCI, Tokyo, Japan, i.p.) 30 minutes before RI exposure. Groups were divided into 3 subgroups based on time of sacrifice $(15,30$, or 90 days post-RI). Experimental animals were administered $1.5 \mu \mathrm{g} / 100 \mathrm{~g}$ of thyroxine and $1 \%$ calcium lactate in drinking water to maintain an euthyroid state.

\section{\begin{tabular}{l|l|l|l|l|l|l|l|l|l}
\hline H & I & G & H & L & I & G & H & T & S \\
\hline
\end{tabular}}

- Salivary functions in the epigallocatechin-3-gallate (EGCG) groups were better than in the radioiodine group.

- EGCG group showed more mucin-rich parenchyma and less periductal fibrosis.

- Fewer apoptotic cells were observed in EGCG group than in radioiodine group.

- EGCG supplementation could protect from radioiodine-induced salivary gland damage.
Measurements of body weights, salivary gland weights, salivary lag times, and salivary flow rates

Mice were weighed and administered ketamine $(100 \mathrm{mg} / \mathrm{kg})$ and xylazine $(5 \mathrm{mg} / \mathrm{kg})$ in sterile water by intraperitoneal injection. A fresh solution of pilocarpine $(0.5 \mathrm{mg} / \mathrm{mL})$ was prepared in phosphate buffered saline, and $0.01-\mathrm{mL} / \mathrm{g}$ body weight i.p. was administered to each mouse. Saliva was collected for 10 minutes after pilocarpine administration with mice positioned vertically (head-down). Salivary lag times were measured and saliva was collected for 10 minutes in preweighed $0.75-\mathrm{mL}$ Eppendorf tubes. Immediately after saliva collection, mice were euthanized by cervical dislocation. Submaxillary glands and tongues were excised.

\section{Morphological analysis of tissues and TUNEL assay}

SGs and tongues were immediately placed in $4 \%$ paraformaldehyde at room temperature, embedded in paraffin, and sectioned at $4 \mu \mathrm{m}$. SGs were stained with alcian blue (AB) and Masson's trichrome (MT), and tongues were stained with hematoxylin and eosin (H\&E).

Apoptosis in submaxillary gland tissues was determined using a terminal deoxynucleotidyl transferase biotin-dUDP nick end labeling (TUNEL) assay using an ApopTag Plus in situ Apoptosis Kit (Chemicon Int., Temecula, CA, USA). TUNEL-positive cells were detected at a magnification of $\times 400$, and numbers of TUNEL-positive cells were counted in 10 random high power fields. TUNEL assays were performed at 15, 30, and 90 days post-RI exposure.

\section{SPECT protocol of animals study}

At 90 days post-RI, technetium pertechnetate $\left(55.5 \mathrm{MBq},{ }^{99 \mathrm{~m}} \mathrm{Tc}\right]$ $\mathrm{TcO}_{4}^{-}$; New Korea Industrial) was administered i.p. to anesthetized mice, which were maintained in an unconscious state during the entire imaging protocol using isoflurane ( 2 volume $\%$ in air). Whole-body single photon emission computed tomography (SPECT) imaging was started immediately after the [ $\left.{ }^{99 \mathrm{~m}} \mathrm{Tc}\right]$ $\mathrm{TcO}_{4}{ }^{-}$injection and repeated every 5 minutes for 100 minutes (NanoSPECT; Bioscan Inc., Washington, DC, USA). Overall, 21 images were obtained per mouse. A fresh solution of pilocarpine $(0.5 \mathrm{mg} / \mathrm{mL})$ was then prepared in phosphate buffered saline, and administered at $0.01 \mathrm{~mL} / \mathrm{g}$ body weight (i.p.), 60 minutes after SPECT.

\section{Whole body SPECT protocol}

Whole body SPECT images were obtained using a large field-ofview rotating gamma camera equipped with four multi-pinhole collimators. The acquisition parameters used were; 24 projections over $360^{\circ}$, circular orbit, and a total acquisition time of 6 minutes (4 seconds per projection). Tomographic images were reconstructed using an iterative reconstruction algorithm $[13,14]$. 


\section{SPECT image analysis}

SPECT images were reviewed and processed using InVivoScope (Bioscan Inc.) and Osirix imaging software (The Osirix Foundation, Geneva, Switzerland). Regions of interest (ROIs) were first drawn manually around thyroid and SGs on images obtained 60 minutes posttreatment that best showed contours of the thyroid and SGs. ROIs of each lesion were combined into a volume of interest (VOI) and VOIs were copied and pasted onto SPECT images, except for images obtained at 60 minutes posttreatment. All VOIs were corrected to ensure they did not contain noise counts from neighboring tissues, such as, bone. The radioactivities of all voxels in VOIs were measured and corrected for activity decay posttreatment. Maximal normalized radioactivity in VOIs were used as representative values to minimize partialvolume effects.

\section{Statistical analysis}

Data analysis was performed using Graph Pad Prism 5 package (GraphPad Software Inc., La Jolla, CA, USA). The significances of differences between groups were evaluated using the KruskalWallis test followed by post hoc testing with Dunn's test. $P$-values of $<0.05$ were considered statistically significant. All results are expressed as means \pm SDs.
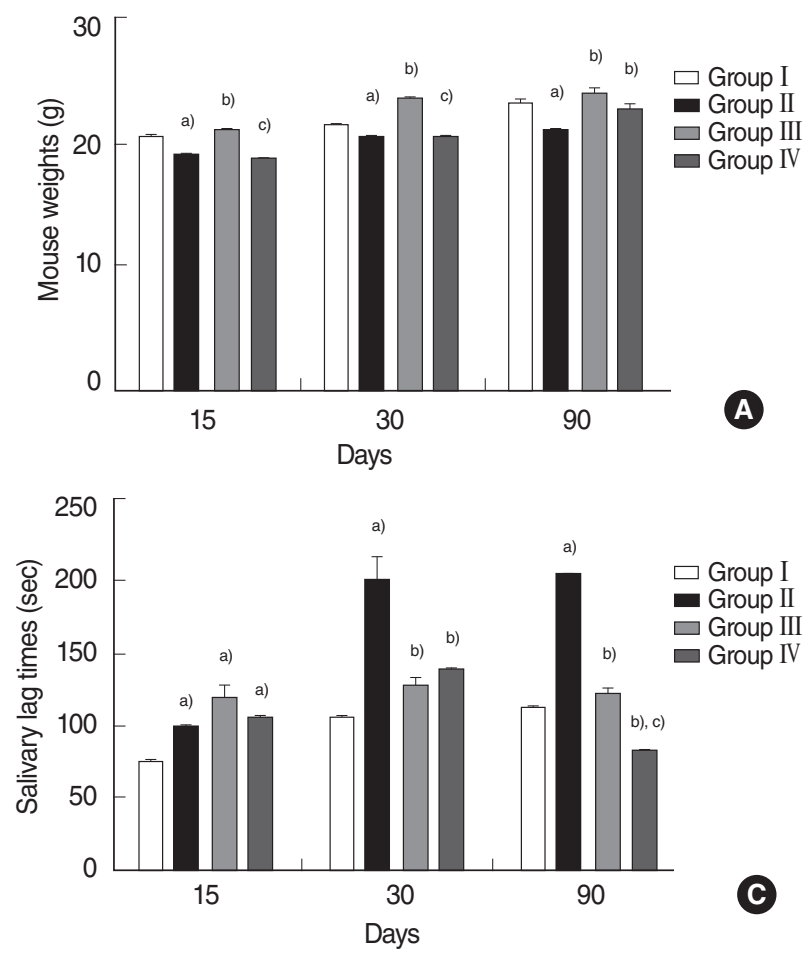

\section{RESULTS}

\section{Changes in body and salivary gland weights}

Before the experiment, no significant weight difference was observed between the all groups $(P>0.05)$. However, at 15, 30, and 90 days posttreatment, mice in the RI group weighed significantly less than mice in the normal control group, and mice in the EGCG group weighed significantly more than mice in the RI group (all $P<0.05$, respectively) (Fig. 1A). Animals in the amifostine group weighed significantly more than animals in the RIexposed group at 90 days post-RI $(P<0.05)$ (Fig. 1A). At 15 and 30 days posttreatment, mice in the EGCG group weighed significantly more than mice in the amifostine group (both $P<0.05$, respectively) (Fig. 1A). SG weights were no different in the 4 groups at 15 and 90 days post-RI (all $P>0.05$, respectively) (Fig. 1B). However, SGs in the normal control weighed significantly more than those in the other 3 groups at 30 days post-RI $(P<0.05)$ (Fig. 1B).

\section{Salivary lag times and salivary flow rates}

No significant intergroup difference was observed for lag times at 15 days post-RI in the RI, EGCG or amifostine group $(P>0.05)$ (Fig. 1C). However, at 30 and 90 days post-RI, lag
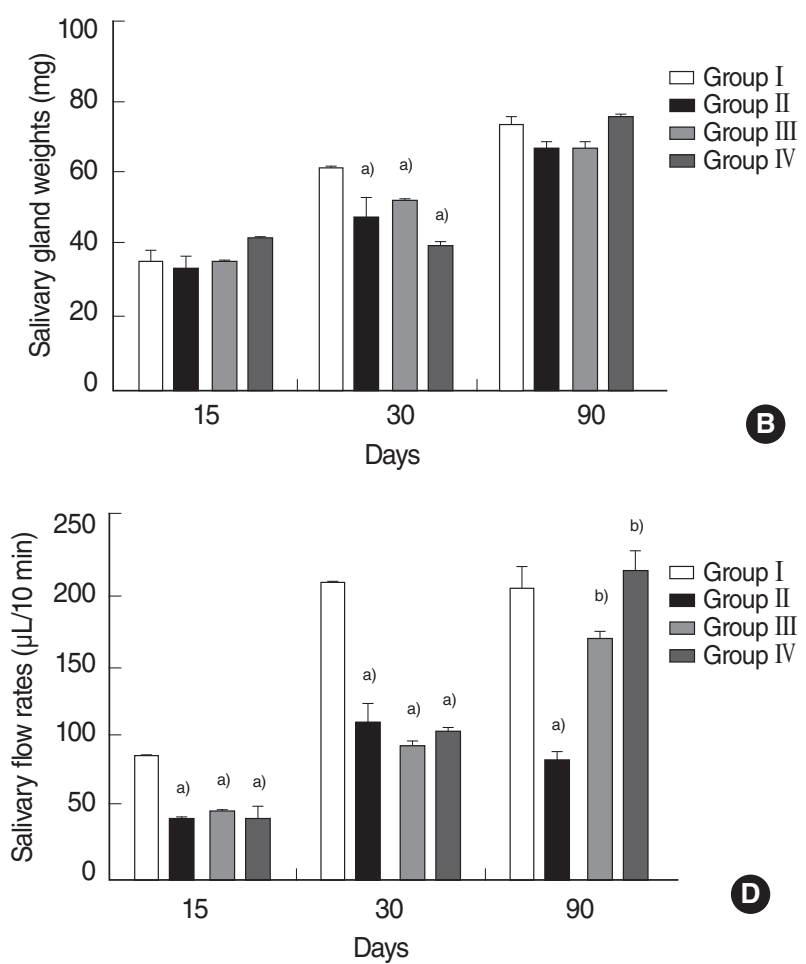

Fig. 1. Comparisons of mouse weights (A), salivary gland weights (B), salivary lag times (C), and flow rates (D). (A) Mice weight in the EGCG or amifostine treated groups was heavier. (B) SG weight in the normal control group was heavier. (C) Lag times and salivary flow rates in the EGCG or amifostine group were shorter and greater respectively. Kruskal-Wallis test and Dunn post hoc multiple comparison test (all $P<0.05$, respectively). Group I, the normal control; group II, RI exposed group; group III, administration of EGCG before RI exposure; group IV, admin-

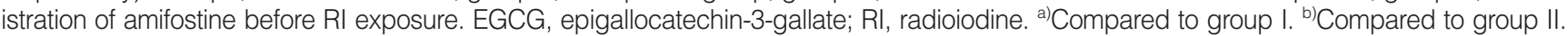
${ }^{c}$ Compared to group III. 
times in the EGCG or amifostine treated group were significantly shorter than in the RI group (all $P<0.05$, respectively) (Fig. 1C). At 90 days, lag times in the amifostine group were significantly shorter than in the EGCG group $(P<0.05)$ (Fig. 1C). In addition, no significant difference between salivary flow rates in the RI, EGCG, and amifostine groups at 15 and 30 days post-RI (all $P>0.05$, respectively) (Fig. 1D). At 90 days post-RI, salivary flow rates in the EGCG or amifostine treated group were greater than in the RI group $(P<0.05)$ (Fig. 1D). There were no significant differences between EGCG and amifostine group at 15, 30, and 90 days post-RI (all $P>0.05$, respectively) (Fig. 1D).

\section{Histological changes and apoptosis}

Microscopic histological changes in SGs were visualized by AB and MT staining at 90 days post-RI. Mucin-containing acini stained with Alcian blue appeared to be more numerous in the EGCG or amifostine group than in the RI group. EGCG or amifostine group also exhibited less periductal and perivascular fibrosis than the RI group (Fig. 2). Filiform papillae of tongues were evaluated by H\&E staining at 90 days post-RI. H\&E staining revealed the RI group showed irregular epithelium on cornified filiform papillae and debris on the tongue surface. However, the EGCG or amifostine group showed uniform, smooth epithe-

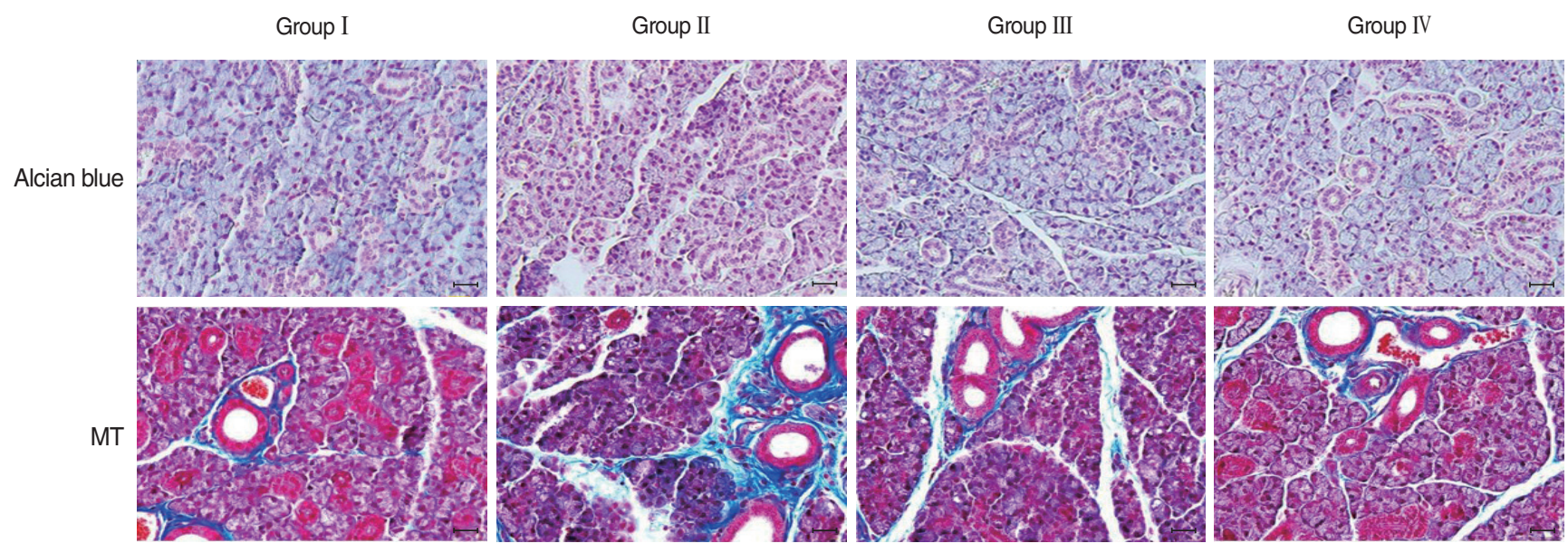

Fig. 2. Histological analysis of salivary glands. Mucin-containing acini stained with Alcian blue appeared to be more numerous in the EGCG or amifostine group than in the RI group. MT staining showed the EGCG or amifostine group exhibited less periductal and perivascular fibrosis than the RI group. Group I, the normal control; group II, RI exposed group; group III, administration of EGCG before RI exposure; group IV, administration of amifostine before RI exposure. EGCG, epigallocatechin-3-gallate; RI, radioiodine; MT, Masson's trichrome (scale bar, 20 «m).
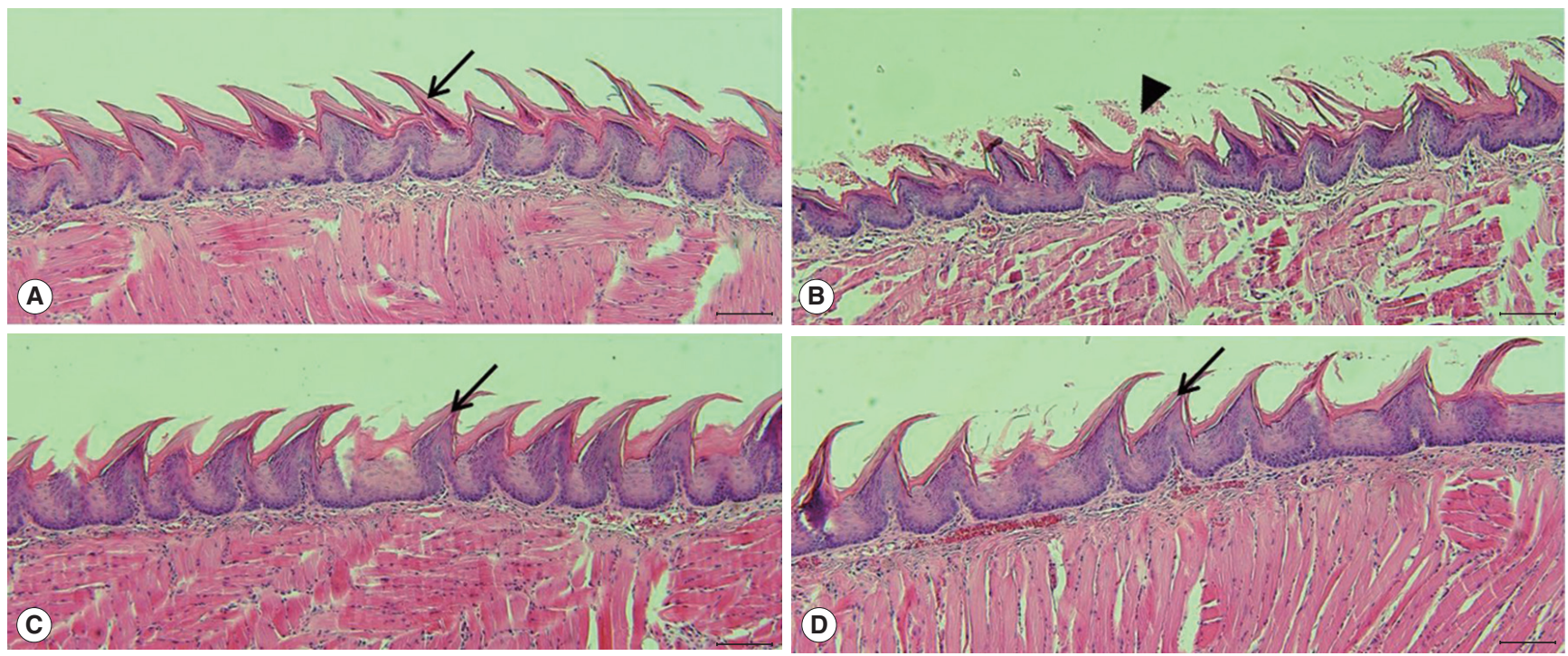

Fig. 3. Histological analysis of tongues. Tongue staining showed irregular epithelium, a cornified layer of filiform papillae, and debris on tongue surfaces in the RI group (arrowhead). However, animals in the EGCG or amifostine group showed uniform, smooth epithelium, and clean tongue surfaces as compared with the RI group (arrow). (A) Group I, the normal control; (B) group II, RI exposed group; (C) group III, administration of EGCG before RI exposure; and (D) group IV, administration of amifostine before RI exposure. EGCG, epigallocatechin-3-gallate; RI, radioiodine (scale bar, $100 \mu \mathrm{m})$. 

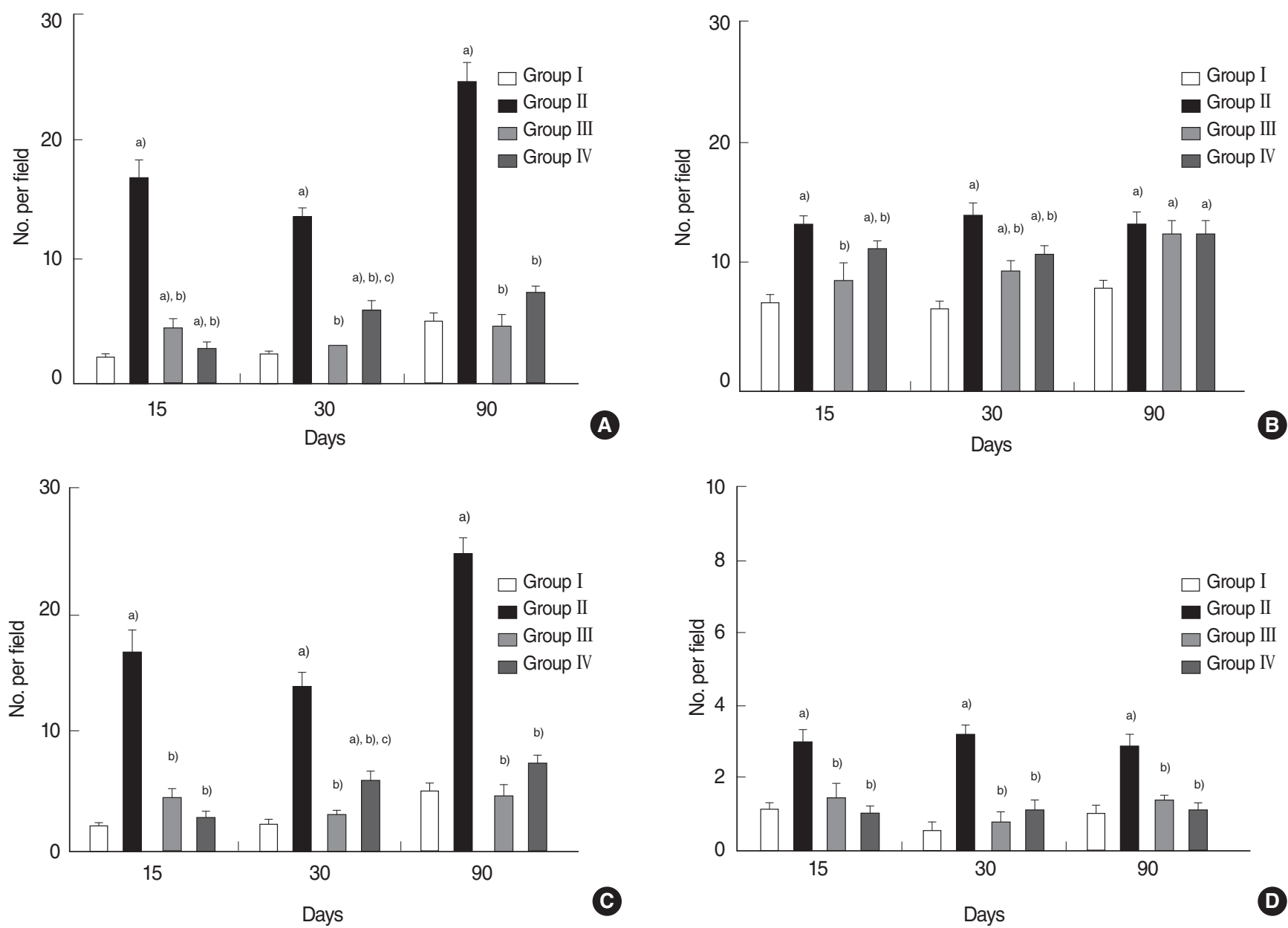

Fig. 4. Quantitative analysis by terminal deoxynucleotidyl transferase biotin-dUDP nick end labeling (TUNEL) assay. (A) Total numbers of TUNEL-positive cells, (B) TUNEL-positive acinar, (C) ductal cells, and (D) endothelial cells. (A) Analysis showed that total numbers of TUNELpositive cells were significantly reduced in the EGCG or amifostine group as compared with RI group. (B-D) Numbers of TUNEL-positive acinar, ductal, and endothelial cells were significantly higher in the RI group and decreased in EGCG or amifostine treated group. Kruskal-Wallis test and Dunn's post hoc multiple comparison test (all $P<0.05$, respectively). Group I, the normal control; group II, RI exposed group; group III, administration of EGCG before RI exposure; group IV, administration of amifostine before RI exposure. EGCG, epigallocatechin-3-gallate; RI, radioiodine. ${ }^{\text {a) }}$ Compared to group I. ${ }^{\text {b) }}$ Compared to group II. ${ }^{c}$ Compared to group III.

lium and no surface debris (Fig. 3).

TUNEL assays showed that numbers of TUNEL-positive cells were significantly higher in the RI group at 15,30 , and 90 days post-RI, and that numbers were significantly lower in the EGCG or amifostine group (all $P<0.05$, respectively) (Fig. 4A). Similarly, numbers of TUNEL-positive acinar, ductal, and endothelial cells were significantly elevated in the RI group but not in the EGCG or amifostine group at 15, 30, and 90 days post-RI (all $P<0.05$, respectively) (Fig. 4B-D).

Dynamics of ${ }^{99 \mathrm{~m}} \mathrm{Tc}$ pertechnetate uptake and excretion

A 90 days post-RI levels of ${ }^{99 \mathrm{~m}} \mathrm{Tc}$ pertechnetate excretion were markedly lower in the RI group, but levels of excretion in the EGCG or amifostine group were similar to that in the normal control group (Fig. 5).

\section{DISCUSSION}

The incidence of thyroid cancer is increasing over the last few decades, and surgery and/or RI ablation therapy was usually performed to treat well differentiated thyroid cancers [15]. RI therapy is an excellent treatment modality for the ablation of residual tumor cells. However, RI affects many nonthyroidal tissues, such as, SGs, lacrimal glands, gastric mucosa, mammary glands, and bone marrow. These tissues all express sodium iodide symporter, which mediates the active transport of iodide ions into cells and thyroid follicular cells in particular [16].

RI-induced sialadenitis occurs in $2 \%$ to $67 \%$ of thyroid cancer patients that have undergone RI therapy [17], and thus, the topic of sialadenitis after RI exposure has received considerable attention. RI has been shown to accumulate 30-40 times more 


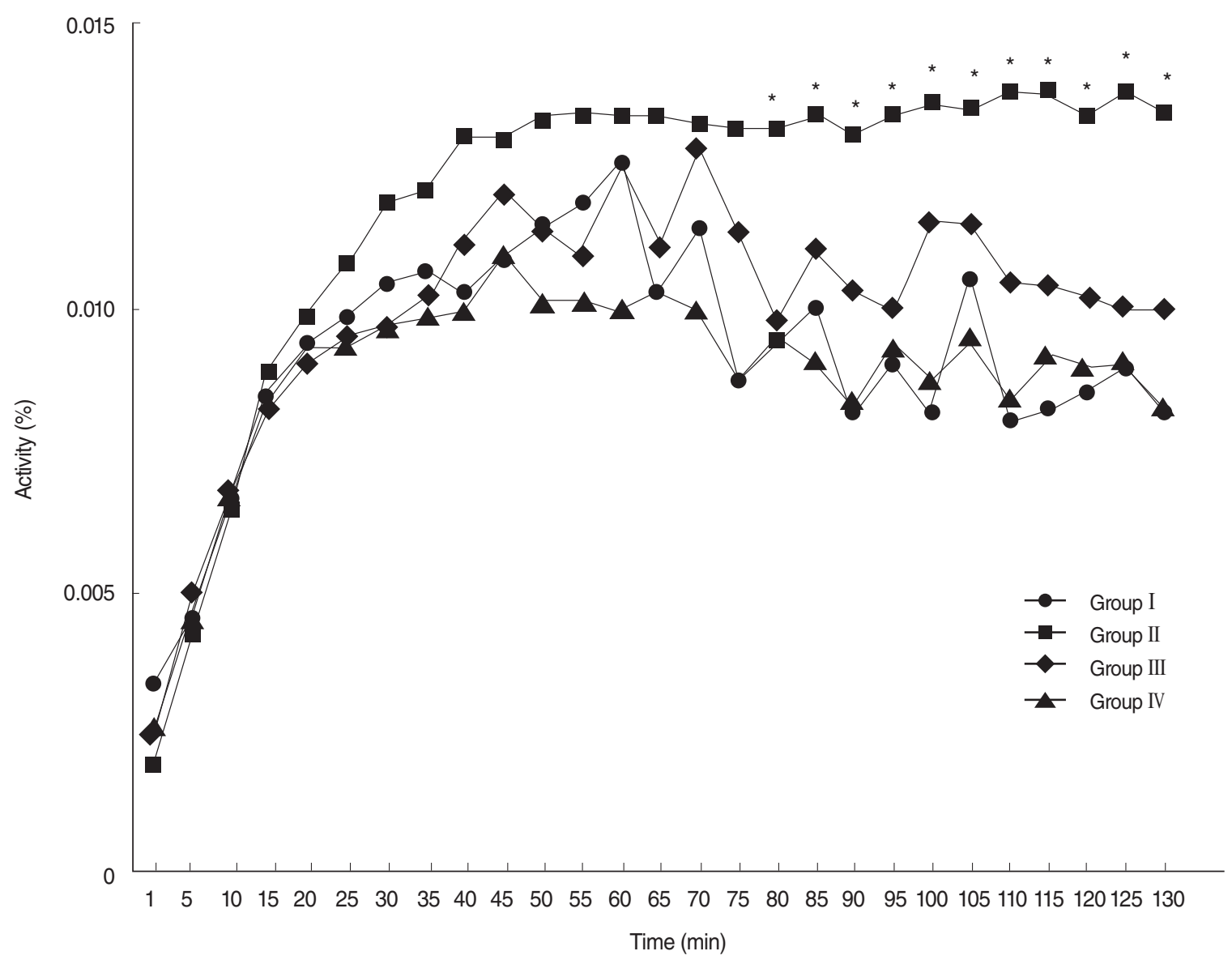

Fig. 5. Dynamics of ${ }^{99 \mathrm{~m}} \mathrm{Tc}$ pertechnetate at 90 days post-RI. At 90 days posttreatment, ${ }^{99 \mathrm{~m}} \mathrm{Tc}$ pertechnetate excretion was markedly lower in the RI-exposed group than other groups, but ${ }^{99 m}$ excretions in the EGCG or amifostine group were similar to that observed in the normal control group. Group I, the normal control; group II, RI exposed group; group III, administration of EGCG before RI exposure; group IV, administration of amifostine before RI exposure. EGCG, epigallocatechin-3-gallate; RI, radioiodine (Asterisks denote significant points).

in SGs than in plasma, and SG dysfunction is one of the important complications of RI therapy and one with serious impacts on patients. RI-induced sialadenitis, which may be transient or permanent, impairs quality of life [18], not only does it cause obstructive sialadenitis and its associated symptoms of swelling and pain, but in the chronic state can also cause hyposalivation. Because patients with differentiated thyroid cancer have a good prognosis, it is critical that the side effects of RI therapy be minimized, and thus, the protection of SGs from RI therapy is an important issue.

In an attempt to prevent the above-mentioned complications, healthy SGs must be protected. The administration of a radioprotective agent prior to radiation to prevent side effects is one such potential preventive measure. In vivo studies on cell protection from radical oxygen species generated by ionizing radiation have been conducted over the years, and amifostine has been shown to have low toxicity and to protective SGs well from RI [19]. Amifostine has also been reported to be organ specific, and is considered to protect normal tissues from the acute and late effects of radiation in head and neck cancer. Fur- thermore, the U.S. Food and Drug Administration (FDA) authorized its use as a radioprotective agent in 1999 [20]. In fact, amifostine is the only drug approved by the FDA for radioprotection in cancer patients. However, it has some adverse effects that can lead to its discontinuation in some patients [21]. In addition, its limited administration route, cost, and the need for medical supervision have limited its clinical use [2,22], and its beneficial effects in patients with thyroid cancer undergoing RI therapy have yet to be established [23]. Hence, the major focus of the present study was to develop a highly effective and nontoxic SG radioprotectant for therapeutic RI exposure.

Many phytochemicals have been shown to have unique abilities to prevent radiation damage [24]. Recent research attention has focused on the protective role of EGCG in epidermal epithelial cells subjected to ultraviolet exposure $[7,8]$, and in these studies, EGCG was found to protect epidermal keratinocytes from ultraviolet induced hydrogen peroxide formation [9]. Dickinson et al. [25] showed that EGCG is able to restore the levels of antioxidant defense enzymes providing protection to the salivary glands from oxidative damage in nonobese diabetic mice 
and human salivary gland cell. Saito et al. [26] also demonstrated that EGCG administration to this murine model protects salivary glands from oxidative stress-induced tissue injury. That is, EGCG-mediated increased expression of antioxidants and reduced expression of autoantigens play an important role in the protection of salivary glands against oxidative stress-induced DNA damage and apoptosis in autoimmune sialadenitis of mice [26].

In the present study, significant intergroup differences in body weights were observed. In particular, animals in the EGCG or amifostine group weighed significantly more than animals in the RI group at 90 days post-RI. SG function importantly influences nutritional intake and general condition, and as a normal body weight usually reflects a healthy status, reductions of body weights in the EGCG or amifostine treated groups implies the radioprotective effect of EGCG or amifostine concerning SGs. Furthermore, lag times were lower and salivary flow rates were significantly higher in the EGCG or amifostine group than in the RI group, which suggests EGCG is as effective as amifostine in terms of protecting against RI-induced SG damage.

RI-exposed SGs showed cellular injury, acinar loss and disorganization, glandular duct enlargement, and marked lipomatosis $[4,27]$. In a previous study, SGs in RI-exposed mice displayed pale cytoplasm, atypical ductal configuration, septal widening, lymphocyte infiltration, cytoplasmic vacuolization with pleomorphism, and elevated levels of tissue fibrosis [28]. In the present study, mice pretreated with EGCG or amifostine showed significant changes in SG architectures at 90 days post-RI. In particular, the EGCG or amifostine group contained higher numbers of mucin containing acini than the RI group. Because mucin has low solubility, high viscosity, elasticity, and lubricity, and adheres well to surfaces, it is an important component of mastication, speech, swallowing, and the removal of bacteria from oral mucosa [29]. The EGCG or amifostine group also exhibited less periductal and perivascular fibrosis than the RI group, and periductal fibrosis causes ductal stiffness and secretion disorders [29]. Accordingly, we believe our observations of increased mucin quantities and reduced ductal fibrosis in EGCG or amifostine group indicate less gland dysfunction. Furthermore, our examination of filiform papillae showed that oral hygiene was better maintained in the EGCG or amifostine group.

Apoptosis is a possible mechanism of RI-induced SG damage. Kutta et al. [27] found higher levels of apoptosis in the SGs of rabbits exposed to RI and observed the radioprotective effect of amifostine. In another study, TUNEL assays revealed more apoptotic cells in both acini and ducts in RI-exposed mice [28]. In the present study, the number of TUNEL-positive cells was significantly higher in the RI-exposed group than in controls, but EGCG or amifostine both reduced this increase. Thus, it appears EGCG or amifostine both blocks the apoptotic cascade.

Joseph et al. [4] also showed that antioxidant (Ocimum sanctum) or amifostine pretreated SGs in rats exhibited greater ${ }^{99 \mathrm{~m}} \mathrm{Tc}$ pertechnetate excretion than an RI alone. In the present study, we investigated spatial distributions of ${ }^{99 \mathrm{~m}} \mathrm{Tc}$ pertechnetate uptake and its excretion from SGs. Levels of ${ }^{99 \mathrm{~m}} \mathrm{Tc}$ pertechnetate excretion were found to be markedly lower in RI-exposed group than in the control group, but excretion levels in the EGCG or amifostine treated groups were similar to in normal controls at 90 days post-RI. These results support the view that EGCG or amifostine relieves ductal distress induced by RI.

Regarding topic worthy of future study, it is evident EGCG could sustain gland function by preventing apoptosis, but the mechanisms responsible for the protection it affords are poorly understood, and thus, further investigations are required to unveil the molecular mode of action of this unique phytochemical. Moreover, although it has been established EGCG induces differential oxidative environments that favor tumor cell destruction and normal cell survival [30], further studies are needed to determine whether EGCG protects thyroid tumor cells.

In the present study, EGCG was found to protect SGs effectively from RI-induced damage, and EGCG was observed to have a beneficial effect on gland histology and functional study and on extents of salivary excretion by SPECT in mice exposed to RI. The protective property of EGCG is believed to involve the reduction of ROS, which is induced by ionizing radiation in SG cells, especially in salivary ductal cells. In the case of differentiated thyroid carcinoma, the use of amifostine as a radioprotectant has not been unequivocally accepted. Controversial reports regarding its efficacy underline the need to identify a better radioprotectant for SGs exposed to high-dose RI therapy. The present study demonstrates the radioprotective effect of EGCG on mouse SGs exposed to RI. We recommend the use of EGCG be explored in detail to determine its effects on SG functions and its potential as a safe radioprotectant for use in patients with differentiated thyroid carcinoma scheduled to undergo RI therapy.

In conclusion, our findings suggest EGCG supplementation before RI therapy could have protective effects similar to amifostine in terms of protecting SGs from RI-induced damage and possibly restoring RI-damaged SG functions.

\section{CONFLICT OF INTEREST}

No potential conflict of interest relevant to this article was reported.

\section{ACKNOWLEDGMENTS}

This research was supported by the Basic Science Research Program maintained by the Korean Society of Head and Neck Surgery, Seoul and by an Inha University Research Grant, Incheon, Republic of Korea. 


\section{REFERENCES}

1. Mandel SJ, Mandel L. Radioactive iodine and the salivary glands. Thyroid. 2003 Mar;13(3):265-71.

2. Weiss JF, Landauer MR. History and development of radiation-protective agents. Int J Radiat Biol. 2009 Jul;85(7):539-73.

3. Okumura H, Nasu M, Yosue T. Effects of amifostine administration prior to irradiation to the submandibular gland in mice: autoradiographic study using 3H-leucine. Okajimas Folia Anat Jpn. 2009 Feb; 85(4):151-60.

4. Joseph LJ, Bhartiya US, Raut YS, Hawaldar RW, Nayak Y, Pawar YP, et al. Radioprotective effect of Ocimum sanctum and amifostine on the salivary gland of rats after therapeutic radioiodine exposure. Cancer Biother Radiopharm. 2011 Dec;26(6):737-43.

5. Ma C, Xie J, Jiang Z, Wang G, Zuo S. Does amifostine have radioprotective effects on salivary glands in high-dose radioactive iodinetreated differentiated thyroid cancer. Eur J Nucl Med Mol Imaging. 2010 Aug;37(9):1778-85.

6. Ma C, Xie J, Chen Q, Wang G, Zuo S. Amifostine for salivary glands in high-dose radioactive iodine treated differentiated thyroid cancer. Cochrane Database Syst Rev. 2009 Oct;(4):CD007956.

7. Katiyar SK. Skin photoprotection by green tea: antioxidant and immunomodulatory effects. Curr Drug Targets Immune Endocr Metabol Disord. 2003 Sep;3(3):234-42.

8. Afaq F, Adhami VM, Ahmad N, Mukhtar H. Inhibition of ultraviolet B-mediated activation of nuclear factor kappaB in normal human epidermal keratinocytes by green tea Constituent (-)-epigallocatechin-3-gallate. Oncogene. 2003 Feb;22(7):1035-44.

9. Katiyar SK, Afaq F, Perez A, Mukhtar H. Green tea polyphenol (-)epigallocatechin-3-gallate treatment of human skin inhibits ultraviolet radiation-induced oxidative stress. Carcinogenesis. 2001 Feb;22 (2):287-94.

10. Mukhtar H, Ahmad N. Tea polyphenols: prevention of cancer and optimizing health. Am J Clin Nutr. 2000 Jun;71(6 Suppl):1698S$1702 \mathrm{~S}$.

11. Sueoka N, Suganuma M, Sueoka E, Okabe S, Matsuyama S, Imai K, et al. A new function of green tea: prevention of lifestyle-related diseases. Ann NYAcad Sci. 2001 Apr;928:274-80.

12. Hsu S, Dickinson DP, Qin H, Lapp C, Lapp D, Borke J, et al. Inhibition of autoantigen expression by (-)-epigallocatechin-3-gallate (the major constituent of green tea) in normal human cells. J Pharmacol ExpTher. 2005 Nov;315(2):805-11.

13. Vanhove C, Defrise M, Franken PR, Everaert H, Deconinck F, Bossuyt A. Interest of the ordered subsets expectation maximization (OS-EM) algorithm in pinhole single-photon emission tomography reconstruction: a phantom study. Eur J Nucl Med. 2000 Feb;27(2):140-6.

14. Defrise M, Vanhove C, Nuyts J. Perturbative refinement of the geometric calibration in pinhole SPECT. IEEE Trans Med Imaging. 2008 Feb;27(2):204-14.

15. La Vecchia C, Malvezzi M, Bosetti C, GaravelloW, Bertuccio P, Levi
F, et al. Thyroid cancer mortality and incidence: a global overview. Int J Cancer. 2015 May;136(9):2187-95.

16. Levy O, De la Vieja A, Carrasco N. The Na+/I- symporter (NIS): recent advances. J Bioenerg Biomembr. 1998 Apr;30(2):195-206.

17. Van Nostrand D. Sialoadenitis secondary to ${ }^{131}$ I therapy for well-differentiated thyroid cancer. Oral Dis. 2011 Mar;17(2):154-61.

18. La Perle KM, Kim DC, Hall NC, Bobbey A, Shen DH, Nagy RS, et al. Modulation of sodium/iodide symporter expression in the salivary gland.Thyroid. 2013 Aug;23(8):1029-36.

19. Dorr RT. Radioprotectants: pharmacology and clinical applications of amifostine. Semin Radiat Oncol. 1998 Oct;8(4 Suppl 1):10-3.

20. Hensley ML, Schuchter LM, Lindley C, Meropol NJ, Cohen GI, Broder G, et al.American Society of Clinical Oncology clinical practice guidelines for the use of chemotherapy and radiotherapy protectants. J Clin Oncol. 1999 Oct;17(10):3333-55.

21. Jensen SB, Pedersen AM, Vissink A, Andersen E, Brown CG, Davies AN, et al. A systematic review of salivary gland hypofunction and xerostomia induced by cancer therapies: management strategies and economic impact. Support Care Cancer. 2010 Aug;18(8):1061-79.

22. Hosseinimehr SJ. Trends in the development of radioprotective agents. Drug DiscovToday. 2007 Oct;12(19-20):794-805.

23. Kim SJ, Choi HY, Kim IJ, Kim YK, Jun S, Nam HY, et al. Limited cytoprotective effects of amifostine in high-dose radioactive iodine 131-treated well-differentiated thyroid cancer patients: analysis of quantitative salivary scan. Thyroid. 2008 Mar;18(3):325-31.

24. Noaparast Z, Hosseinimehr SJ. Radioprotective agents for the prevention of side effects induced by radioiodine-131 therapy. Future Oncol. 2013 Aug;9(8):1145-59.

25. Dickinson D, DeRossi S, Yu H, Thomas C, Kragor C, Paquin B, et al. Epigallocatechin-3-gallate modulates anti-oxidant defense enzyme expression in murine submandibular and pancreatic exocrine gland cells and human HSG cells. Autoimmunity. 2014 May;47(3):177-84.

26. Saito K, Mori S, Date F, Ono M. Epigallocatechin gallate inhibits oxidative stress-induced DNA damage and apoptosis in MRL-Fas(lpr) mice with autoimmune sialadenitis via upregulation of heme oxygenase-1 and Bcl-2.Autoimmunity. 2014 Feb;47(1):13-22.

27. Kutta H, Kampen U, Sagowski C, Brenner W, Bohuslavizki KH, Paulsen F.Amifostine is a potent radioprotector of salivary glands in radioiodine therapy. Structural and ultrastructural findings. Strahlenther Onkol. 2005 Apr;181(4):237-45.

28. Choi JS, Park IS, Kim SK, Lim JY, Kim YM. Morphometric and functional changes of salivary gland dysfunction after radioactive iodine ablation in a murine model. Thyroid. 2013 Nov;23(11):1445-51.

29. Choi JS, Park IS, Kim SK, Lim JY, Kim YM. Analysis of age-related changes in the functional morphologies of salivary glands in mice. Arch Oral Biol. 2013 Nov;58(11):1635-42.

30. Yamamoto T, Hsu S, Lewis J, Wataha J, Dickinson D, Singh B, et al. Green tea polyphenol causes differential oxidative environments in tumor versus normal epithelial cells. J Pharmacol Exp Ther. 2003 Oct;307(1):230-6. 